Objectives This study aimed to assess whether peripheral monocytes in RA are pre-programmed to become M1 proinflammatory macrophages.

Methods Blood was collected from healthy donors, at-risk individuals (Those with arthralgia, $\mathrm{ACPA}+/ \mathrm{RF}+$, normal CRP and no evidence of synovitis) and established RA patients. CD14+ monocytes were isolated from peripheral blood mononuclear cells using a CD14 magnetic bead separation kit. Cells were stimulated with LPS $(100 \mathrm{ng} / \mathrm{ml})$ for 3-24 hours and to assess the effects of STAT3 inhibition, cells were pretreated with STATTIC $(10 \mu \mathrm{M})$ for 30mins. A Human Cytokine and Chemokine PCR array was carried out and those genes most differentially expression were further validated in a larger cohort of patients using RT-PCR. The metabolic profile of cells was analysed using Seahorse XFE Technology, which concomitantly analysis glycolysis and mitochondrial respiration in real-time. Gene and protein expression of key inflammatory and glycolytic markers was also carried out by RT-PCR, western blotting and ELISA.

Results CD14+ RA monocytes are hyper-inflammatory upon stimulation, with significantly higher expression of IL-1 $\beta$, TNFo, IL-6, IL-27, CXCL10 and CXCL11 compared to healthy controls, which is indicative of a M1-like pro-inflammatory phenotype. These hyper-inflammatory cells are highly glycolytic, with increased expression of $\mathrm{HIF} 1 \alpha, \mathrm{HK}$ and PFKFB3, key glycolytic enzymes. Both baseline glycolysis and baseline oxidative phosphorylation are increased in RA CD14 + monocytes, paralleled by increased ATP synthesis and maximal respiratory capacity, suggesting a hyper-energetic phenotype. This hyper-inflammatory, hyper-glycolytic phenotype is mediated by STAT3, as selective STAT3 inhibition can significantly decrease M1-like cytokines and PFKFB3 and HK2 expression. In addition, STAT3 inhibition significantly decreases both oxidative phosphorylation and glycolysis pathways. Finally, this pro-inflammatory phenotype in evident in $\mathrm{CD} 14+$ monocytes from arthralgia $\mathrm{ACPA}+\mathrm{RF}+$ people at risk of developing disease, demonstrating that these processes may precede clinical manifestations in RA.

Conclusions This study demonstrates the unique inflammatory and metabolic phenotype of RA monocytes, suggesting that peripheral $\mathrm{CD} 14+$ monocytes may be pre-programmed to become M1-like pro-inflammatory macrophages. In addition, the observation of this phenotype in at-risk individuals indicates that these features may precede clinical manifestations of RA and therefore could be useful as a biomarker for early diagnosis.

Disclosure of Interest None declared.

\section{P069 CONSTITUTIVE OVEREXPRESSION OF INTERLEUKIN 38 HAS A NEGATIVE IMPACT ON HUMAN NHK KERATINOCYTE FITNESS}

L Mermoud*, D Talabot-Ayer, N Brembilla, J Julia Borowczyk-Michalowska, G Palmer. Department of Pathology-Immunology, University of Geneva School of Medicine, Geneva, Switzerland

\subsection{6/annrheumdis-2018-EWRR2019.58}

Career situation of first and presenting author Student for a master or a $\mathrm{PhD}$.

Introduction Interleukin (IL)-38 is a member of the IL-1 cytokine family. Based on its sequence homology with the IL-1 and IL-36 receptor antagonists, IL-38 was proposed to act as an anti-inflammatory molecule. However, few data are available to date about its biological role and mechanism of action. Highest constitutive IL-38 expression is detected in the skin, where the cytokine is produced mainly by differentiated keratinocytes.

Objectives The aim of this study is to investigate the function of IL-38 in human keratinocytes, using the immortalized normal human keratinocyte (NHK) cell line stably transfected with vectors allowing for constitutive or inducible IL-38 overexpression.

Methods NHK cell proliferation and mortality were assessed respectively by cell counting and by measuring $\mathrm{LDH}$ activity in the supernatant of high density cell culture. Differentiation of NHK cells was initiated by switching to a culture medium containing high $\mathrm{Ca}^{++}$and serum. mRNA levels of differentiating NHK cells were determined by RTqPCR.

Results Constitutive IL-38 overexpression reduced the proliferation of NHK cells and lead to increased mortality. Differentiating NHK cells constitutively overexpressing IL-38 had increased mRNA levels for the early differentiation marker keratin 10 as compared to empty vector transfected control cells. On the contrary, expression of the late differentiation marker involucrin was reduced in NHK cells constitutively overexpressing IL-38. To further explore potential effects of IL-38 on keratinocyte differentiation, while circumventing the impact of its constitutive overexpression on cell fitness, we set up a doxycyclin-inducible Tet-On system to overexpress IL-38 in NHK cells.

Conclusions We are currently optimizing 2D and 3D culture conditions for in vitro differentiation of NHK cells to mimic more closely the situation in human skin. We will then study the impact of IL-38 on NHK cell differentiation using cells transfected with the inducible IL-38 expression system. In conclusion, our results suggest that constitutive overexpression of IL-38 decreases proliferation and viability of NHK cells. We will thus use an inducible expression system to further investigate the effects of IL-38 on NHK cells.

Disclosure of Interest None declared.

\section{P070/009 MIR-221-3P IS UPREGULATED IN RHEUMATOID ARTHRITIS AND AFFECTS JAK3/STAT3 SIGNALING IN M2-MACROPHAGES}

${ }^{1,2} \mathrm{~L}$ Quero*, ${ }^{*}, 2 \mathrm{E}$ Hanser, ${ }^{1,2} \mathrm{AN}$ Tiaden, ${ }^{1,2} \mathrm{D}$ Kyburz. ${ }^{1} D B M$, University of Basel; ${ }^{2}$ Experimental Rheumatology, University Hospital, Basel, Switzerland

10.1136/annrheumdis-2018-EWRR2019.59

Career situation of first and presenting author Post-doctoral fellow.

Introduction Recurrent activation of synovial macrophages is considered as intrinsic factor that precedes rheumatoid arthritis (RA)-related autonomous cytokine cascades. microRNAs (miRs) and Toll-like receptors (TLRs) play a pivotal role in regulating the inflammatory response in innate immune cells and are at dysregulated levels in RA patients.

Objectives Here we explored TLR-regulated miRs that tune the inflammatory response of M2-type macrophages.

Methods Based on a miR-screen in TLR-stimulated human M2-macrophages we selected miR-221-3p to analyze its expression profile in clinical samples from RA, other inflammatory arthritis (OIA), osteoarthritis (OA) and healthy donors (HD) by qPCR. We challenged M1- and M2-macrophages with elevated miR-221-3p and/or miR-155-5p expression 